\title{
Aspectos Clínicos y Hematológicos Iniciales de la Leucemia en el Niño
}

\author{
Dres. Laularo Vargas P. ${ }^{1-2-3}$. Sylvia Pino R. ${ }^{1-2}$, Marcela Barría C. ${ }^{2-4}$, \\ Teresa Yourte $\Lambda^{1-2}$. Hugo Gatría $\mathrm{l}, 1 \cdot 2 \cdot 3$
}

\section{Clinical and Hematological Features in Acute Leukemia}

\begin{abstract}
The initial clinical and hemathological features of 94 paticnts with acute leukemia are analyzed. These childxen were studied in the Hoxpital San Juar de Dios of Santiago de Chile between 1964 and 1978. The lymphoblastic type was predominant, with $82 \%$ of the cases. The peak incidence was observed betseen 2 and 6 years of age. Four syndromes were observed in this disease: anemic, purpuric, febrile and intiltrative tumoral and occured un vasiable combinations in almost all our cases $(93,6 \%$. Only $6,4 \%$ of patients had one syndrome alonc at the moment of the diagnosis. The aleukemic leukemia was very infrequent, being noticed in only 5 patients, two of them with an uplistic syndrome rceluiring up to 4 bone marrow aspirations in order to confirm the diagnosis of leukemia. The diagnosis in the remaining patients was certified by the first bone marrow differential count.
\end{abstract}

En el momento del diagnóstico de la Leucemia Aguda la proliferación blástiça ha alcanzado una masa considerable como lo demuestran los estudios de cinética celular, tanto clínicos como experimentales $^{1-2}$.

Si bien los blastos leucémicos se originan en los órganos hemato y/o linfopoyéticos, una característica de estas células es proliferar e invadir cualquier tejido u órgano. Ultimamente se ha cumprobado que algunos tipos de leucemia, especialmente las leucemias a linfoblastos $T$ se caracterizan por un compromiso extramedular más precoz y acentuado ${ }^{3-4}$.

Estas consideraciones explican lo protciforme de las manifestaciones clínicas iniciales en algunos pacientes.

El compromiso medular en la Leucemia $\mathrm{Agu-}$ da es una constante $y$ el uso rutinario del hemograma y del mielograma por aspiración, que son los exámenes que permiten el diagnóstico de la Leucemia Aguda, explican que éste se realice sin mayores dificultades.

Sin embargo, la Leucemia Aguda en su inicio puede simular o coexistir con patologias muy

1 Servicio de Pediatría Hospital San Juan de Dios.

2 Departamento de Pediatria. División Ciencias Médicas Occidente, Facultad de Medicina Universidad de Chile.

3 GOPEC'H: Grupo Oncológico Pediátrico Chileno.

4 Departamento de Salud Pública. División Ciencias Médicas Occidente, Facultad de Medicina Universidad de Chile.

Presentado en Congreso Becarios Pediatría Concepción, Noviembre 1980. diversas. El no tener en cuenta algunos síntomas orientadores (púrpura, adenopatias, anemia, etc.), puede retardar el diagnóstico en algunos enfermos, to cual tiene importancia para el pronóstico, ya que una leucenia diagnosticada tardíamente por lo general presenta complicaciones de tipo infeccioso (sepsis), o hemorragias.

De ahi nuestro interés en revisar la sintomatología inicial de los pacientes estudiados en nuestro Servicio entre 1964 y 1978 con el propósito de determinar la frecuencia de los diferentes signos y sintomas de la Leucemia Aguda cuyas combinaciones permitan una sospecha diagnóstica oportuna.

\section{MATERIAL Y METODO}

Se analizan en forma retrospectiva las características clínicas y hematológicas de presentación de 94 pacientes con Leucemia Aguda diagnosticada en el Hospital San Juan de Dios entre 1964 y 1978. Se consideraron solamente los casos debidamente comprobados con nielograma.

En la catalogación de la casuística se empleó la clasificación morfológica tradicional: Linfoblástica (que incluye las leucemias llamadas por otros Hemocitoblásticas), Mieloblástica, Promielocitica y Mielomonocitica.

La morfología citológica fue estudiada en todos los casos por uno de nosotros (L.V.), empleándose también tinción panóptica de May Gruwald Giemsa. En algunos casos dudosos se emplearon tinciones adicionales (PAS, peroxidasas) y se requirió la opinión de otros citólogos. 


\section{RESULTADOS}

Distribución según Tipo Citológico: En mestra casuística se observó un franco predoninio de la Leucemia Aguda Linfoblástica (L.A.L.), que rcpresentó un $82 \%$ de los casos (77 pacientes).

Del $18 \%$ restante, aproximadamente la nitad correspondió a Leucenuia Aguda Micloblástica (L.A.M.), y la otra mitad a Leucenua Aguda Mielomonocitica (L.A.MM.) y Leucemia Promiclocítica (LPM). No se observaron casos de Liritroleucemia.

Al igual que en otros estudios no encontramos diferencias significativas entre tipo citológico y sintomatología inicial por lo cual el análisis posterior corresponde a todos los tasos ${ }^{5-5}$.

Distribución por Edad y Sexo. (Figura 1). Al igual que to descrito en la literatura nacional y extranjera ${ }^{5} \cdot 6 \cdot 7 \cdot 8$ hemos encontrado und mayor concentración de casos en la edad preescolar. No observanos diferencias significativas en la distribución por sexo.

Lugar de Procedencia. Antes de realizar esta revision teniamos la impresión de que existía una mayor concentración de casos en algunas comu. thas rurales que correspondian en esc entonces il Area Occidente. Sin embargo, el análisis de las tasas de incidencia según población no demostró diferencias significativas entre las comunas.

Diagnóstico Pediátrico Inicial: Si bien como se analiza más adelante, el diagnóstico de leucemia aguda no ofrecc dudas en la gran mayoría de los casos no ocume lo mismo cuando el pediatra enfrenta al enfermo por primera vez. En la casuística examinada los enlermos llegaron al Hospital o al Consultorio de Hematologít sólo en el $42,6 \%$ con el diagnostico de sospecha de Leucenvia Aguda.
Ln un $19,1 \%$ adicional se sospechó una enfermedad hematológica (anemia o púrpura). En el $38.3 \%$ restante los diagnósticos variaron entre estado infeccioso, enfermedad reumática, artritis reumatoidea juvenil, fiebre tifoidea, sepsis de origen desconocido, etc.

Manifestaciones Clinicas: En la figura 2 aparecen los síntomas de presentación de nuestra casuística. La palidez, la fiebre $y$ el púrpura en orden decreciente constituyeron los sintomas predominantes que indujeron al enfermo a consultar. Con menos frecuencia nit obscrvaron los dolores useos y la epistaxis.

Los diversos síntomas de la leucemía aguda pueden agruparse en cuatro grandes sindromes: anémico, purpúrico, febril y tumoral o infiltrativo. Fstos cuatro sindromes generalmente coexisten y se combinan entre sí de diferente manera en cada enfermo.

E.l sindrome anénico (palide $\%$, fatigabilidad, disnea de esfuerzo, palpitaciones. hemoglobina bajo $11 \mathrm{gr} \%$ ) es el mas frecuente y se confirma con el laboratorio en un $94 \%$. Se debe destacar que se trata generalmentc de und anemia de instalación relativamente lenta y progresiva, frecucntemente bién tolerada excepto en los enfermos que presentaban hemorragias importantes $y$ recientes. En el $16 \%$ de los pacientes la anemia era muy intersa con menos de $10 \%$ de hematocrto lo que determinaba que existieron sintomas de descompensación hemodinámica. Algunos llegaron con un cuadro de insuficiencia cardítica manifiesta.

El sindrome purpirico (petequias, equimosis. gingivorragias, epistaxis y otras hemorragias) sigue en frecuencia $(70,2 \%)$ y a veces es muy leve $y$ pasit inadvertido para los padres o sólo es demostrable por el laboratorio con el liallazgo de

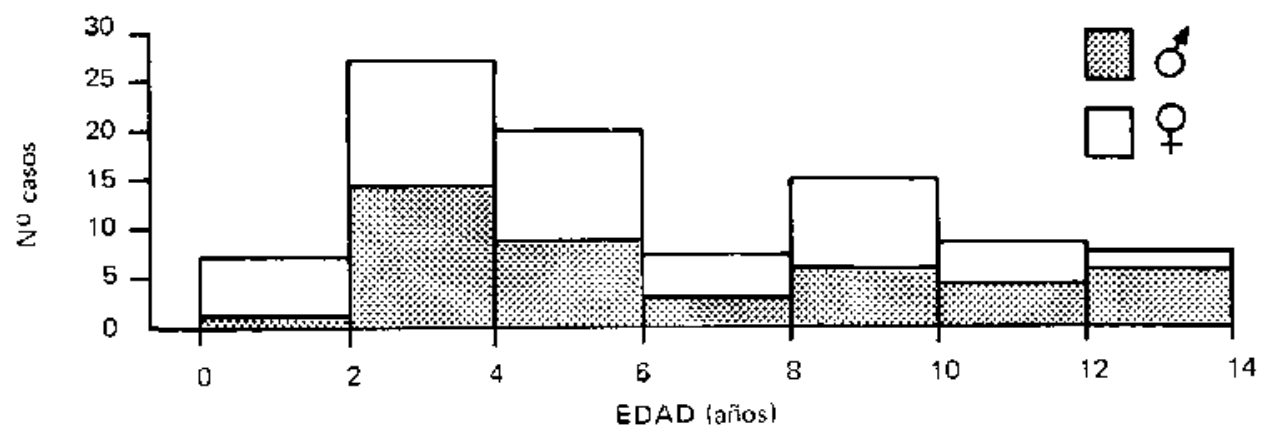

Figura 1.

Distribución por lidad de 94 Casos do Leucemia Aguda 


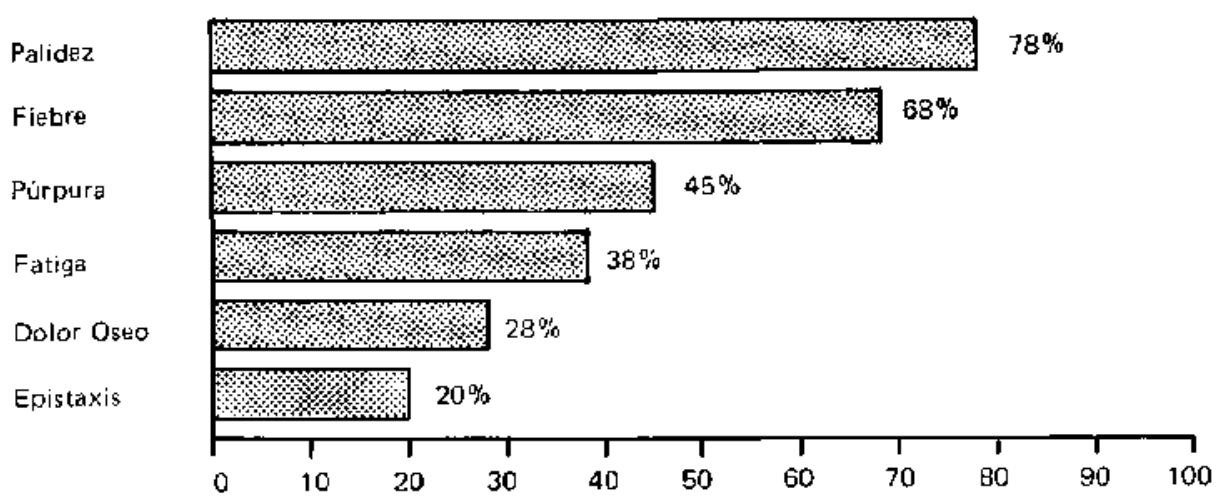

Figura 2.

Sínturaa de Presentación en 94 Casc : de' Leucemia Aguda

trombocitopenia $(14,9 \%)$. La hemorragia más frecuente después de la cutanea os la epistaxis siendo excepcional la hemorragia digestiva o urinaria.

En más de la mitad do los casos $(56,4 \%)$ se presentó un síndrome febril que en alrededor del $50 \%$ obedecía a una complicación infecciosa.

Cabe señalar que sólo el $50 \%$ de los casos tenia un sindrome infiltrativo tumoral expresado en hepatomegalia, esplenomegalia o ambas (más de tres centimetros bajo el reborde costal) y adenopatías menores de $1 \mathrm{~cm}$. Un $28,7 \%$ presentaban dolores óseos importantes. Sólo un $1,1 \%$ tenía al inicio un sindrome de hipertensión endocraneana debido a infiltración meníngea.

La gran mayoría de los enfermos presentaba una combinación de los síndromes analizados. En más de los $2 / 3$ de los casos se observó la suma de síndrome anémico y purpúrico acompañandose además de fiebre o de hepatoesplenomegalia o adenomegalias tumorales.

La combinación de los $\mathbf{4}$ síndromes determina que el diagnóstico clínico sea cusi seguro. Cuando se combinan 3 de estos sindromes la sospecha clínica de leucemia es muy alta. Estas dos situa. ciones las observamos en el 77,6\% de los casos. En el $16 \%$ de los casos solo se presentaron dos síndromes: sindrome anémico y sindrome febril o purpúrico. Las formas incompletas mono sindromáticas son más raras y sólo las comprobamos en el $6,4 \%$ de nuestra serie ( 6 casos).

Estudio Hernatológico Inicial: La sospecha diagnóstica se confirmó con el estudio hematológico que demostró la presencia de blastos en cantidad significativa (más de1 $15 \%$ ) en el $67 \%$ de los niños (Tabla 1), destacándose que 14 de ellos tenían blastemia superior a $50.000 / \mathrm{mm}^{3} .31$ pacientes se presentaron inicialmente con escaso porcentaje de blastos y en 5 de ellos no se observaron estas células en el hemograma inicial, el ler. mielo- grana resolvió el diagnóstico en 29 de estos niños. al demostrar más de un $40 \%$ de infiltración blástica y sólo en 2 pacientes fue necesario repetirlo varias veces ya que se trataba de Leucemia Aguda de forma aplástica, sin visceromegalia.

Tabla I.

Presencia de Blastos en el Hemograma Inicial de 94 Casos de Leuemia Aguda.

\begin{tabular}{|c|c|c|c|}
\hline F'orma de Leucemia & $\begin{array}{l}\text { \%ode } \\
\text { Blastos }\end{array}$ & $\begin{array}{l}\mathrm{N}^{\circ} \mathrm{de} \\
\text { Casos }\end{array}$ & $\%$ \\
\hline Leucemiza & & & \\
\hline Alellcénica & 0 & 5 & 5,3 \\
\hline Leucenia & & & \\
\hline $\begin{array}{l}\text { Subleucémica } \\
\text { Leucernia }\end{array}$ & $1-5$ & 12 & 12,8 \\
\hline Paucileucémica & $6 \cdot 15$ & 14 & 14,9 \\
\hline Sub-Total & & 31 & 33,0 \\
\hline $\begin{array}{l}\text { Leutemia } \\
\text { Leucémica }\end{array}$ & $>15$ & 63 & 67.0 \\
\hline TOTAL & & 94 & 100,0 \\
\hline
\end{tabular}

En cuanto a la serie roja (Tabla 2), la mayoría de los enfermos ( $85 \%$ ) presentó anemia de cierta consideración destacíndose que en el $16 \%$ de los niños el hematocrito era menor de $10 \%$. Sólo un $3,2 \%$ tuvo valores normales.

En la Tabla 3 aparece la distribución de los casos según número de leucocitos. En el $31 \%$ había leucopenia, mientras que las formas hiperleucocitaria correspondieron a un $25,5 \%$.

En relación a las plaquetas (Tabla 4) encontramos que el $75 \%$ de los casos presentó una trombocitopenia que fue muy acentuada en $1 / 3$ de ellos. Es necesario destacar que un $25,6 \%$ de 
los pacientes tuvo más de 100.000 plaquetas por $\mathrm{mm}^{3}$.

Tabla 2.

Distribución de 94 Casos de Leucemial Apudi serrín Henatusitu Inicial.

\begin{tabular}{lrr} 
Hematocito & No & $\%$ \\
& & $\% \%$ \\
$11 \quad 30 \%$ & 15 & 16.0 \\
$30 \quad 34 \%$ & 65 & 69,1 \\
$350 \mathrm{~min} \%$ & 11 & 11,7 \\
\hline TOTAL & 3 & 3.2 \\
\hline
\end{tabular}

Tabla 3.

Distribución de 94 Casos de Lenesmia Aguda según $N^{0}$ de Leuexitos e'n Hemoggramia lnicial.

\begin{tabular}{|c|c|c|}
\hline Letcocitos $; \mathrm{mm}^{3}$ & 10 & $\%$ \\
\hline$\cdot 4.000$ & 29 & 30.9 \\
\hline $4.001 \quad 10.000$ & 23 & 24.5 \\
\hline 30.000 & 18 & 19.1 \\
\hline Más de 30.000 & 24 & 25.5 \\
\hline
\end{tabular}

Tubia 4

Distribución de 94 Cusos de lescemia Agudi seguin Recuento de Plaquetas en Hemograma Iricial.

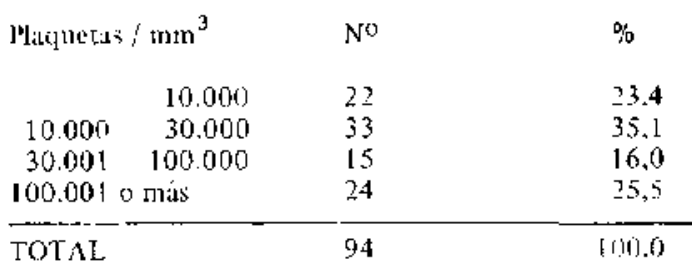

\section{DISCUSION}

Los signos y sintomas de la Leucemia Aguda se pueden explicar a través de dos mecanismos fisiopatológicos:

Insufleiencia medular: Anemia, granulocitopenia que condiciona la fiebre y/o las infecciones $y$ trombecitopenia que produce purpura $y$;o hemorragias de mucosas. La insuficiencia medular se debe a la invasión blástica de la médula y a la acción inhibitoria que ejercen los blastos en el tejido hematopoyético normal.

Proliferación blastica que explica las adenonegalias, la hepatoesplenomegalia, los dolores óseos $y$ las alteraciones radiológicas del esqueleto.

$\mathrm{El}$ análisis de nuestra casuistica demuestra que en la mayoría de los casos existe combinación de ambos fenómenos ya sea en forma completa o frustra, así, observamos que el 93,6\% de ellos presentó formas clínicas polisindromáticas y sólo el $6.4 \%$ hizo un sindrome aislado: anémico, febril, purpúrico o infiltrativo.

El hemograma también expresa en la gran mayoria de los casos una combinación de signos de insuficjencia medular tanemia arregenerativa. neutropenia y/o trombocitopenia) con signos de proliferación tumoral (presencia de blastos en sangre periférica).

Sin embargo, es importante hacer notat que un número no despreciable de casos no tiene trombucitopenia inicialnente $(25,5 \%)$.

La ausencia de anemia es mucho menos frecuente $(3,2 \%)$. pero en un $11,7 \%$ ésta es muy leve y podria no dársele la importancia necesaria.

La lalta de blastos en el henograma inicial es también un hecho intrabitual. sólo un $5,5 \% \mathrm{co}$ menzó como Jeucemia aleucémica y es importante destacar que en 12,8\% de los enfermos los blastos exan escasos en el hemograma (entre 1 y $5 \%$ ): sin embargo en la mayoría de ellos la presencia de anemia y de trombocitopeniá obligó a practicar el mielograma que permitió resolver $\mathrm{el}$ problema diagnóstico.

Sólo de 7 de nuestrus pacientes se presentaron como Leucemias Aleucémicas de format aplástica o con mielofibrosis' situación que es dilícil de ditcrenciar de una Aplasia Medular propiamente tal. El diagnóstico diferencial entre estas dos entidades se logra mediante mielogramas repetidos o biopsia nedular. Excepcionalmente, cuando lid duda persiste, algunos alutores recomiendan el uso de esteroides que producen una respuesta rapida si lit pancitopenia es debidal a leucemia lo que no ocurre si es por aplasid 10

Los otros 3 casos de Leucemia Alcutérnica tenian visceromegalia que hacia nuy sospechoso el diagnóstico, el que tue confimado por el mielograma que demostró una invasion de blastos significativa. En 2 de estos elfermos cxistía pancitopenia periférica.

No es el proposito de la presente revisión analizar los problemas de clasificacion citológica de la Leucemia $\Lambda$ guda, que sc presentan con alguna frecuencia. Para ellos es necesario la realización de técnicas citoquímicas complementarias como tambiér el examen del frotis por otros citólogos. Actualmente la clasificación Franco Americano Británica (F.A.B.) ${ }^{11}$ permite en la mayoria de los casos clasificar las leucemias desde el punto de vista citológico en forma más objetiva.

El análisis de nuestra casuistica nos pernite afirmar que es posible plantear la sospecha clinica de Leucemia Aguda en la gran mayoría de los casus. 
Como es una enfermedad relativamente rara se piensa poco en ella, asi en los casos febriles se hace el djagnóstico de fjebre tifoidea, enferme. dad mucho más frecuente en nuestro medio o cuadros sépticos de otro origen; en aquellos en que predomina el púrpura se plantea el diagnóstico de púrpura Trombocitopénico Idiopático $y$ por último si lo predominante son los dolores óseos se piensa primero en Enfermedad Reumáti. ca O Artritis Reumatoídea Juyenil ${ }^{12 \cdot 13}$. Sin embargo, una mayor acuciosidad en la historia y en el examen físico permitiría una sospecha diagnóstica más precoz que se confirmaría con el estudio hematológico.

En la literatura se mencionan los siguientes cuadros susceptibles de confundirse con Leucemia Aguda:

Monomucleosis infecciosa o síndrome mono. nucleósico, especialmente aquellos casos que evolucionan con trombocitopenia. Sin embargo, la citología de los linfoblastos es notoriamente distinta de la morfología de los linfocitos atípicos virales. Las reacciones serológicas (Paul Bunnel o Monotest) constituyen una valiosa ayuda en el điagnóstico diferencial.

Sindrome mieloproliferutivos y sindrome pre. leucémicos. Son muy infrecuentes en -el niño ${ }^{14}$. En estos cuadros se observa anemia refractaria, trombocitopenia, neutropenia, hepatoesplenomegalia y en la médula ósea hay hiperplasia mieloide $y$ eritroide. Frecuentemente se encuentra reacción leucoeritroblástica y monocitosis que rara vez se observan en la Leucemia Aguda.

Reaccion Leucemoide. Se puede observar en infecciones graves (sepsis) en hemólisis aguda, vasculitis y Tuberculosis miliar, etc. En la Leuce. mia Aguda Granulocítica existen células inmaduras (mieloblastos y/o promielocitos) y granulocitos maduros (polinucleares segmentados) sin que aparezcan las células intermedias (mielocitos y juveniles), fenómeno denominado hiatus leucémico que no se ve en la reacción leucemoide.

Sepsis con neutropenia. Algunos casos de sepsis grave se acompañan de neutropenia y en el mielograma muchas veces se encuentra una acumulación de células inmaduras especialmente promielocitos lo que podría inducir al diagnóstico de Leucemia Promielocitica.

El tratamiento antibiótico resuelve el problema ya que al mejorar la sepsis también lo hace la neutropenia y desaparece la detención de la maduración de los promielocitos en la médula ósea.

Ncuroblastoma. La morfología de los neuroblastos es muy similar a la de los linfoblastos, de ahí que los raros casos de neuroblastoma con invasión medular y neuroblastos en sangre perifé- rica pueden fácilmente confundirse con Leucenuia Linfoblástica. El diagnóstico se puede aclarar al encontrarse una tumoración mediatinal posterior o una masa suprarrenal calcificada, que son hallazgos propios del Neuroblastoma. La determinación de catecolaminas es una valiosa ayuda, como también la formación de rosetas en la médula, los que no se observan en la leucemia.

En nuestra casuistica no se presentó el problema de diagnóstico diferencial con los cuadros precedentes.

\section{RESUMEN}

Se analizan las caracteristicas clinicas y hematológicas iniciales de 94 pacientes (44 niños y 50 niftas) con leucemia aguda tratados en el Hospital San Juan de Dios entre 1964 y 1978.

Se observó un franco predominio de los preescolares. El tipo citológico predominante correspondió a leucemia aguda linfoblástica ( $82 \% .77$ casos). La forma polisindromática se presentó en e1 $93,6 \%$ de los casos ( 88 paciente) con combina. çión variable de los cuatro síndromes característicos de esta enfermedad: anémico, purpúrico, febril e infiltrativo. Sólo un $6.4 \%$ tenía un sólo síndrome presente en el momento del diagnóstico. La leucemia aleucémica es de muy baja frecuencia observándose sólo en 5 pacientes. Dos casos de leucemia aleucémica presentaron un cuadro clínico y de laboratorio inicial de aplasia medular lo que produjo problemas diagnósticos que se resolvieron practicando mielogramas sucesivos. En el resto de los casos el mielograma inicial confirmo el diagnóstico que habia sido planteado por los hallazgos clinicos y del hemograma.

\section{REFERENCIAS}

1 Mover A.M.: Cell kinetics and practical consequences for therapy of leukemia N. Engl. J. Med. 293: 389 , 1975

${ }^{2}$ Frei E, Sallon $S$.: Acute lymphoblastic leukemia treatment. Cancer 42:828, 1978 .

${ }^{3}$ Jocquillar CI., Le Gall E., Schaison G., Auclerc M.F. Chastong $C l$. Frondrin $G$.: Leucemies aigues avec localisation thymique. Actuatites Hematologiques 12: $44,1978$.

4 Sallan S., Weinstein H.: Childhood leukemia in "Hematology of Infancy and Childhood". Editado por Nathan D., Oski F.A.W.B. Saunders Co pág. 979, 1981.

5 Bermard J., Bessis $M$ : Hematologie Clinique, Masson et Cie. 1958.

6 Pierce M.I., Borges W.H., Heyn R.M., Wolff J.A., Gilbet E.S.: Epidemiological factors and survival experience in 1770 children with acute leukemia. Cancer 23: $1296,1969$. 
${ }^{7}$ Dameshek W., Guiz F.: Leukemia. Grune and Stratton. New York, 1964

- Vildosole J.: Leukemia Aguda. Lecciones de Pediatría Clínica y Social. Editado por J. Meneghello Ed. Universitaria Santiago Chile. 1965.

${ }^{9}$ Hown I.M., Evans D.I.K., Bone marrow fibrosis in acute lymphoblastic leukemia of childhood J. Clin. Pathol. 31: 313, 1978.

10 Methorn D.K., Gross S.: Acuite childhood leukemia presenting as aplastic anemia: the response to corticosteroide J, Pediatr. 77:647, 1970.
1 I Bennet J.M., Catoysky D., Daniel M.T., Flondrin G. Gatton D.A.G., Gralnick H.R., Suiton C.: Propposals for the classification of the acute leukemia Brit. J. Haematol. 33: 451, 1976.

12 Thomos L.B., Forkner C.E.: The skeletal lesions of acute leukemia. Cancer 14: 608, 1961.

13 Schaller $J$.: Arthritis as a presenting manifestation of malignancy in children J. Pediatr. 81: 793, 1973.

14 Greenberg P., Mara $B$ : the myeloproliferative disorders. Am. J. Med. 61: 878, 1976. 